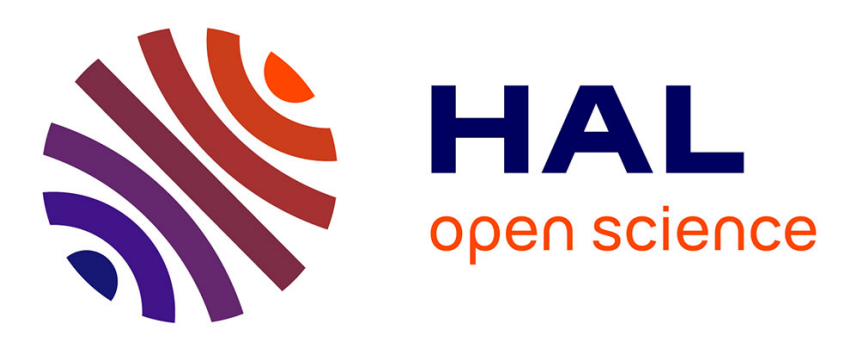

\title{
Response of composite plates in air-backed and water-backed conditions subjected to a far-field underwater explosion
}

Ye Pyae Sone Oo, Hervé Le Sourne, Olivier Dorival

\section{- To cite this version:}

Ye Pyae Sone Oo, Hervé Le Sourne, Olivier Dorival. Response of composite plates in air-backed and water-backed conditions subjected to a far-field underwater explosion. 8th International Conference on Marine Structures (MARSTRUCT 2021), Jun 2021, Trondheim, Norway. hal-03357844

\section{HAL Id: hal-03357844 \\ https://hal.science/hal-03357844}

Submitted on 29 Sep 2021

HAL is a multi-disciplinary open access archive for the deposit and dissemination of scientific research documents, whether they are published or not. The documents may come from teaching and research institutions in France or abroad, or from public or private research centers.
L'archive ouverte pluridisciplinaire HAL, est destinée au dépôt et à la diffusion de documents scientifiques de niveau recherche, publiés ou non, émanant des établissements d'enseignement et de recherche français ou étrangers, des laboratoires publics ou privés. 


\title{
Response of composite plates in air-backed and water-backed conditions subjected to a far-field underwater explosion
}

\author{
Y.P. Sone $\mathrm{Oo}^{1,2} \&$ H. Le Sourne ${ }^{1,2}$ \\ ${ }^{1}$ GeM - Institute of Research in Civil and Mechanical Engineering, UMR CNRS 6183, Ecole Centrale de \\ Nantes, France. \\ ${ }^{2}$ Dept. of Mechanical Engineering, Institut Catholique d'Arts et Métiers (ICAM), Nantes campus, France.
}

\author{
O. Dorival ${ }^{3,4}$ \\ ${ }^{3}$ Clément Ader Institute, UMR CNRS 5312, Toulouse, France. \\ ${ }^{4}$ Dept. of Mechanical Engineering, Institut Catholique d'Arts et Métiers (ICAM), Toulouse campus, France.
}

\begin{abstract}
A semi-analytical design tool is introduced for a rapid analysis of the interaction of a plane shock wave generated by a far-field underwater explosion and the submerged orthotropic plate in both air-backed and water-backed conditions. An efficient numerical scheme called a Nonstandard Finite Difference (NSFD) is adapted to solve the coupled fluid-structure equations involving the first-order Doubly Asymptotic Approximation $\left(\mathrm{DAA}_{1}\right)$ and the geometric nonlinear structural equations. The First-order Shear Deformation Theory (FSDT) is considered along with the simply-supported boundary condition for the orthotropic rectangular plate. The applicability of one-to-one approximation, which is used to simplify the nonlinear mode-coupled equations, is investigated. The semi-analytical approach is then validated by comparing with LS-DYNA/USA (DAA $)_{1}$ results for three case studies: rigid plate-spring system, deformable steel and composite plates subjected to a plane shock wave. Finally, the advantages as well as the limitations of the present approach are discussed.
\end{abstract}

\section{INTRODUCTION}

\subsection{Motivations \& objective}

Consequences of underwater explosions (UNDEX) and the damage they could bring forth have been well understood through meticulous efforts of the past researchers, for example, (Hall 1989; Keil 1961; Office of Naval Research 1950) and so on. Yet, the advancement in the manufacturing techniques and the discovery of novel materials further propelled the need for more research in the domain of the composite structural responses under intense loads such as impacts, in-air or underwater blasts (Mouritz et al. 2001; Mouritz 2017; Porfiri and Gupta 2009). In an attempt to comprehend such dynamic behavior, full-scale or model scale experiments have been conducted, for instance, (Arora et al. 2017; Dear et al. 2017). However, performing experiments on such a large scale could be both costly and time consuming and so, advanced numerical techniques have become popular in recent years (Mair 1999; Sigrist 2015). These studies contain applications of numerical simulations and at the same time, validations by the experiments. One such commonly used numerical code is LS-DYNA which can be coupled to either the acoustic volume element proposed by (Felippa and DeRuntz 1984; Newton 1978), or the Underwater Shock Analysis (USA) code (DeRuntz 1989). These numerical codes are quite powerful, and able to capture complicated phenomena such as shock wave propagation, fluid-structure interaction, cavitation, etc. Nevertheless, the time and effort necessary for both preparation and computation can still be immense. According to the study of (Barras 2012), tools such as LS-DYNA/USA are not wellsuited for the preliminary design studies in which different materials, plate sizes, and load cases need to be considered. In this context, a semi-analytical design tool has been developed within the framework of French research project SUCCESS. One of the objectives of this project is to be able to rapidly analyze the air and underwater blast responses of rectangular composite plates, including the post-damage stage. Practical applications include designing the composite sonar domes, acoustic window of a submarine, and the sailing boat hull.

Keeping this objective in mind, this paper is intended to present analytical and numerical perspectives in regard to the response of simply-supported composite plates subjected to a far-field underwater explosion. The development of semi-analytical formulae comprises coupling of the nonlinear structural equation with the first-order Doubly Asymptotic Approximation $\left(\mathrm{DAA}_{1}\right)$ proposed by (Geers 1978$)$. The composite plate is considered in both air-backed and water-backed conditions. Geometric nonlinearity due to large deflection, in von-Karman sense, is taken into account by incorporating a quadratic strain-displacement relationship. The First-order Shear Deformation Theory (FSDT) and the one-to-one approximation 
approach found in (Nishawala 2011) are adapted to reduce the mathematical complexities arisen. The governing equations that include the Fluid-Structure Interaction (FSI) effect are then discretized and solved by using a Nonstandard Finite Difference (NSFD) numerical scheme developed in (Songolo and Bidégaray-Fesquet 2018). The obtained results are confronted to the numerical solutions using LSDYNA/USA (DAA 1 ). Details of the mathematical development are explained in Section 3.

\subsection{Underwater explosion}

Underwater explosions not only generate a primary shock wave, which propagates through the surrounding fluid medium, but also cause the formation and oscillation of a gas bubble (Cole 1948). Depending on the standoff distance and the charge mass, these can be characterized as near-field or far-field explosions. A far-field underwater explosion is defined as an UNDEX where the charge is located at a sufficient distance away from the structure such that the pressure can be regarded as a plane shock wave and the secondary pressure wave caused by the bubble pulsation can be ignored (Klenow and Brown 2010).

According to (Cole 1948), the plane shock pressure wave can be approximated as:

$P(t)=P_{0} e^{-t / \tau}$, for $0 \leq t \leq \tau$

where $P_{0}$ is the peak pressure, $t$ is the time variable, and $\tau$ is the decay time required for the peak pressure to fall to $1 / e$ of its peak value. The corresponding peak pressure $P_{0}$ and the decay time $\tau$ can be determined from the charge mass $C$, standoff distance $R$ and the type of the explosive charge by using the Principle of Similarity as follows (Cole 1948):

$P_{0}=K_{1}\left(\frac{C^{1 / 3}}{R}\right)^{A_{1}}, \tau=K_{2} C^{1 / 3}\left(\frac{C^{1 / 3}}{R}\right)^{A_{2}}$

where $K_{1}, A_{1}, K_{2}$ and $A_{2}$ are constants that depend on the types of the explosives (e.g., TNT, HBX-1).

When a plane shock wave arrives at the target structure, the total pressure at the interface can be obtained by a linear superposition of the incident and scattered pressures. The scattered pressure involves reflection of the incident pressure after the impact and damping radiation caused by the sudden movement of the plate. This high frequency or early-time interaction phenomenon had been proposed by (Taylor 1941) in which the plate is either free-standing or supported by a linear spring. The late-time phenomenon or the cavitation effect, however, was not considered. The former effect can be associated to an additional pressure created during the plate deceleration while the latter should be accounted for when the total pressure in the fluid drops below the vapor pressure.

Due to its effectiveness, Taylor's formulation has been adapted and advanced by many researchers. For example, the air-backed condition of Taylor's theory has been extended by (Liu and Young 2008) to the water-backed condition. (Schiffer and Tagarielli 2012) also studied the 1D underwater blast response of the rigid plate, taking into account the cavitation. (Hoo Fatt and Sirivolu 2017) extended the approach of Taylor to a 2D deformable plate in both air and water-backed conditions. (Brochard et al. 2018, 2020; Sone Oo et al. 2019) considered a two-step approach: in step I, the underwater blast is idealized as an impulsive loading to account for the early-time response while in step II, the water-added mass is considered as an additional pressure load. However, (Sone Oo et al. 2020), based on various case studies, later pointed out that the impulse-based approach using Taylor's 1D theory is valid only for a certain range of FSI parameter. (Sone Oo et al. 2020b), in this regard, improved the impulse-based formulations by coupling the first-order Doubly Asymptotic Approximation $\left(\mathrm{DAA}_{1}\right)$ to the linear structural equation of the plate, ensuring a smooth transition from the early to the long time phase. However, only a linear solution with an air-backed condition has been considered in that paper. Therefore, this paper serves as an improvement to the previous version by including geometric nonlinearity (in von-Karman sense), and then by extending it to the water-backed condition. The results are analyzed for the spring-supported rigid plate, simplysupported steel and carbon fiber/epoxy plates in airand water-backed conditions, and then compared with numerical solutions using LS-DYNA/USA (DAA 1 ).

\subsection{LS-DYNA/USA}

LS-DYNA/USA is the coupled FSI solver comprising of nonlinear FE code and the Underwater Shock Analysis (USA) code. It determines the transient response of a wholly or partially submerged structure subjected to a shock wave of arbitrary pressure profile (DeRuntz 1989). Application of LSDYNA/USA for naval structures can be found in (Shin 2004; Le Sourne et al. 2003). The fluid equations treated by USA are based on boundary element codes called $\mathrm{DAA}_{1}$ or $\mathrm{DAA}_{2}$, the first- and second-order differential equations in time domain to calculate the fluid pressure due to scattered waves respectively. They take into account both early- and long-time structural motions as well as a smooth transition between the two asymptotes. DAA-based solvers do not require explicit fluid modelling and thus, save computation time. The second-order approximation $\left(\mathrm{DAA}_{2}\right)$ is a generalization of $\mathrm{DAA}_{1}$ with an enhanced accuracy. However, due to the increased complexity of $\mathrm{DAA}_{2}$, only $\mathrm{DAA}_{1}$ is adapted in this paper.

\section{PROBLEM DESCRIPTION}

Two types of problem are studied here: (1) springsupported rigid plate (Figure 1), and (2) a simply- 
supported rectangular plate (Figure 2). The plate has the sides $a, b$ and uniform thickness $h$. A standard Cartesian coordinate system is defined at the origin and mid-surface of the plate. Displacements in $x, y, z$ directions are denoted as $u, v, w$ respectively. A uniformly distributed pressure is considered on one side of the plate. The plates are either in air-backed or water-backed conditions. Cavitation, hydrostatic pressure and structural damping are not considered.

(a)

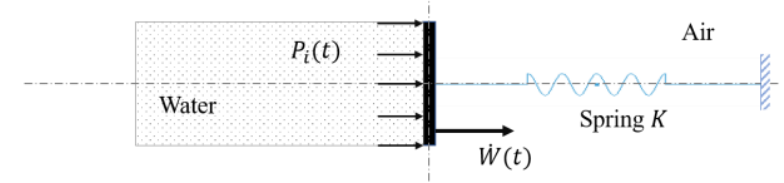

(b)

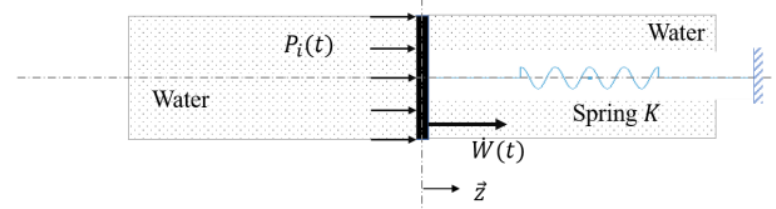

Figure 1. A mass-spring system containing a rigid plate subjected to an incident pressure in: (a) air-backed, and (b) water-backed conditions.

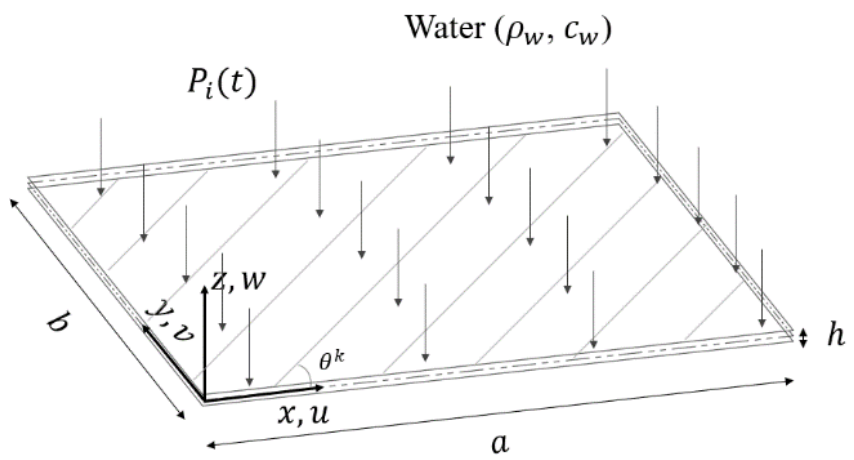

Figure 2. Cartesian coordinate system and geometry of the 2D deformable rectangular plate

\section{ANALYTICAL MODEL}

\subsection{Spring-supported rigid plate}

For a rigid plate with an areal mass $m_{s}$ subjected to a plane shock wave $P_{i}$, the equation of motion can be given as:

$\ddot{W}(t)+\omega^{2} W(t)=\frac{P_{t o t}(t)}{m_{s}}$

where $m_{s}=\rho_{s} h$ is the areal mass of the plate; $\omega=$ $\sqrt{K / m_{S}}$ is the angular frequency of the plate, $K$ is the areal stiffness of the spring, $P_{t o t}(t)$ is the total pressure acting on the plate, and $W(t)$ is the displacement of the plate (positive in $z$-direction). The total pressure $P_{\text {tot }}(t)$ can be obtained by linear superposition of all the pressures as:
$P_{\text {tot }}(t)=P_{i}(t)+P_{s_{L}}(t)-P_{s_{R}}(t)$

where $P_{i}$ is the incident pressure, $P_{S_{L}}$ and $P_{S_{R}}$ are the scattered pressures on the left and right sides of the plate respectively, see Figure 1. Physically, the total pressure is the net pressure acting on the plate.

The scattered pressures can be determined using $\mathrm{DAA}_{1}$ formulations (Geers 1978) as:

$\dot{P}_{s}(t)+D_{f} P_{s}(t)=\rho_{w} c_{w} \dot{u}_{s}(t)$

where $D_{f}=\left(\rho_{w} c_{w}\right) /\left(M_{f}\right), M_{f}$ is the areal wateradded mass for the rigid plate when it moves in water and $\dot{u}_{s}(t)$ is the acceleration of the fluid particle due to scattered wave. Note that Eq. (5) only asymptotically satisfies the conditions in high and low frequencies.

Using the velocity continuity condition at the fluid-structure interface, the scattered wave acceleration can be given as:

$$
\dot{u}_{s}^{L}(t)=\dot{u}_{i}(t)-\ddot{W}(t)
$$

and

$\dot{u}_{S}^{R}(t)=\ddot{W}(t)$

for the left and right sides of the plate respectively.

Equations (3) and (5) together form a system of coupled differential equations for the FSI and can be solved using NSFD method, see the complete derivations in (Sone Oo et al. 2020b, 2021). The time interval $\left[t_{0}, t\right]$ is discretized as:

$t^{i}=t_{0}+(i-1) \Delta t$

where $\Delta t$ is the step size $(\Delta t>0), t_{0}$ is the initial time, $t^{i}$ is the current time step, and $i=1,2,3, \ldots$ refers to the discrete points in time.

The discretized NSFD solutions are as follows:

$$
\begin{aligned}
& W^{i+1}=W^{i} \cos (\omega \Delta t)+V^{i}\left(\frac{\sin (\omega \Delta t)}{\omega}\right)-P_{t o t}^{i}\left(\frac{\cos (\omega \Delta t)-1}{m_{s} \omega^{2}}\right) \\
& V^{i+1}=-W^{i} \omega \sin (\omega \Delta t)+V^{i} \cos (\omega \Delta t)+P_{t o t}^{i}\left(\frac{\sin (\omega \Delta t)}{m_{s} \omega}\right)
\end{aligned}
$$

where $W$ and $V$ are displacement and velocity of the plate respectively.

Let $\varrho$ be the tracing constant to switch on/off the water-backed condition. Then, the scattered pressure for the left side of the plate:

$P_{S_{L}}^{i+1}=P_{S_{L}}^{i} e^{-D_{f} \Delta t}+\left(\frac{1-e^{-D_{f} \Delta t}}{D_{f}}\right)\left(-\rho_{w} c_{w} \dot{V}^{i}+\dot{P}_{i}^{i}\right)$

and for the right side:

$P_{S_{R}}^{i+1}=\varrho\left[P_{S_{R}}^{i} e^{-D_{f} \Delta t}+\left(\frac{1-e^{-D_{f} \Delta t}}{D_{f}}\right)\left(\rho_{w} c_{w} \dot{V}^{i}\right)\right]$

When $\varrho=0, P_{S_{R}}$ will be zero on the right side, representing the air-backed condition. When $\varrho=1$, waterbacked condition is invoked.

The initial conditions are taken as $W(0)=$ $V(0)=0, \dot{V}(0)=2 P_{0} / m_{S}$ and $P_{S_{L}}(0)=P_{i}(0)=P_{0}$, and $P_{S_{R}}=0$. 


\subsection{Simply-supported rectangular plate}

\subsubsection{In-air response}

In this subsection, the First-order Shear Deformation Theory (FSDT) for the orthotropic plate (Figure 2) is extended to account for the geometric nonlinearity due to large deflection. The derivation procedures follow those of (Mei and Prasad 1989) where the equilibrium equations containing the Airy's stress function $\phi$ are considered.

According to FSDT, the transverse displacement is assumed to be independent of the thickness and the transverse normal strain is taken as zero. Only three DOFs are considered, assuming that $|u|,|v| \ll|w|$. The following quadratic strain-displacement relations are applied (Kármán 1907):

$\epsilon_{x x}=\frac{1}{2}\left(\frac{\partial w}{\partial x}\right)^{2}+z \frac{\partial \psi_{x}}{\partial x}, \quad \epsilon_{y y}=\frac{1}{2}\left(\frac{\partial w}{\partial y}\right)^{2}+z \frac{\partial \psi_{y}}{\partial y}$

$\epsilon_{z z}=0, \quad \gamma_{x y}=\left(\frac{\partial w}{\partial x} \frac{\partial w}{\partial y}\right)+z\left(\frac{\partial \psi_{x}}{\partial y}+\frac{\partial \psi_{y}}{\partial x}\right)$

$\gamma_{x z}=\frac{\partial w}{\partial x}+\psi_{x}, \quad \gamma_{y z}=\frac{\partial w}{\partial y}+\psi_{y}$

Equilibrium conditions state the following governing equations:

$\frac{\partial N_{x}}{\partial x}+\frac{\partial N_{x y}}{\partial y}=I_{2} \ddot{\psi}_{x}$

$\frac{\partial N_{x y}}{\partial x}+\frac{\partial N_{y}}{\partial y}=I_{2} \ddot{\psi}_{y}$

$\frac{\partial Q_{x}}{\partial x}+\frac{\partial Q_{y}}{\partial y}+q+q^{*}=I_{1} \ddot{w}$

$\frac{\partial M_{x}}{\partial x}+\frac{\partial M_{x y}}{\partial y}-Q_{x}=I_{3} \ddot{\psi}_{x}$

$\frac{\partial M_{x y}}{\partial x}+\frac{\partial M_{y}}{\partial y}-Q_{y}=I_{3} \ddot{\psi}_{y}$

where $\left(I_{1}, I_{2}, I_{3}\right)=\int_{-h / 2}^{h / 2} \rho\left(1, z, z^{2}\right) d z$ are mass, coupling and rotatory inertia where $I_{2}, I_{3} \approx 0,\left(N_{x}, N_{y}, N_{x y}\right)=$ $\int_{-h / 2}^{h / 2}\left(\sigma_{x x}, \sigma_{y y}, \sigma_{x y}\right) d z$ are in-plane force resultants, $\left(M_{x}, M_{y}, M_{x y}\right)=\int_{-h / 2}^{h / 2} z\left(\sigma_{x x}, \sigma_{y y}, \sigma_{x y}\right) d z$ are bending moment resultants, $\left(Q_{x}, Q_{y}\right)=\int_{-h / 2}^{h / 2}\left(\sigma_{x z}, \sigma_{y z}\right) d z$ are shear force resultants, $q$ is the external force in normal direction, and $q^{*}$ is the resultant transverse force due to membrane effects defined as:

$q^{*}=\frac{\partial^{2} \phi}{\partial y^{2}} \frac{\partial^{2} w}{\partial x^{2}}+\frac{\partial^{2} \phi}{\partial x^{2}} \frac{\partial^{2} w}{\partial y^{2}}-2 \frac{\partial^{2} \phi}{\partial x \partial y} \frac{\partial^{2} w}{\partial x \partial y}$

By taking the work of (Mei and Prasad 1989), it is possible to derive the following equation of motion:

$\rho h \mathcal{N}(\ddot{w})-U(w)=\mathcal{N}(q)+\mathcal{N}\left(q^{*}\right)$

where:

$\mathcal{N}=l_{1} \frac{\partial^{4}}{\partial x^{4}}+l_{2} \frac{\partial^{4}}{\partial x^{3} \partial y}+l_{3} \frac{\partial^{4}}{\partial x^{2} \partial y^{2}}+l_{4} \frac{\partial^{4}}{\partial x \partial y^{3}}+l_{5} \frac{\partial^{4}}{\partial y^{4}}+$

$l_{6} \frac{\partial^{2}}{\partial x^{2}}+l_{7} \frac{\partial^{2}}{\partial x \partial y}+l_{8} \frac{\partial^{2}}{\partial y^{2}}-1$

is a differential operator in which $l_{1}, l_{2}, \ldots, l_{8}$ are coefficients given in Appendix, Eq. (A1). Then:
$\mathcal{U}(w)=\bar{D}(w)+\bar{V}(w)$

is an operator containing variable $w . \bar{D}$ and $\bar{V}$ refer to bending and transverse shear differential operators which can be defined respectively as:

$\bar{D}=D_{11} \frac{\partial^{4}}{\partial x^{4}}+4 D_{16} \frac{\partial^{4}}{\partial x^{3} \partial y}+2\left(D_{12}+2 D_{66}\right) \frac{\partial^{4}}{\partial x^{2} \partial y^{2}}+$

$4 D_{26} \frac{\partial^{4}}{\partial x \partial y^{3}}+D_{22} \frac{\partial^{4}}{\partial y^{4}}$

and:

$\bar{V}=\sum_{j=1}^{7} v_{j} \frac{\partial^{6}}{\partial x^{(7-j)} \partial y^{(j-1)}}$

where coefficients $v_{j}=[\bar{s}]\left[D_{126}\right]$ are defined in Eq. (A4 - A5) of the Appendix section.

To have a closed mathematical problem, a second equation would be required and is obtained from St. Venant's compatibility relation given below:

$A_{11}^{*} \frac{\partial^{4} \phi}{\partial y^{4}}+\left(2 A_{12}^{*}+A_{66}^{*}\right) \frac{\partial^{4} \phi}{\partial x^{2} \partial y^{2}}+A_{22}^{*} \frac{\partial^{4} \phi}{\partial x^{4}}-2\left(A_{16}^{*} \frac{\partial^{4} \phi}{\partial x \partial y^{3}}+\right.$

$\left.A_{26}^{*} \frac{\partial^{4} \phi}{\partial x^{3} \partial y}\right)=\left(\frac{\partial^{2} w}{\partial x \partial y}\right)^{2}-\left(\frac{\partial^{2} w}{\partial x^{2}} \frac{\partial^{2} w}{\partial y^{2}}\right)$

where $A_{i j}^{*}=\left[A_{i j}\right]^{-1}$ in which $(i, j)=(1,2,6)$ for extension, and $(i, j)=(4,5)$ for shear. It should be noted that the right-hand side of Eq. (20) represents the Gaussian curvature, which is zero for developable surfaces, e.g., a cylinder or a cone.

For a simply-supported boundary rectangular plate, solution functions can be expanded into double Fourier series as follows:

$w(x, y, t)=\sum_{m=1}^{\infty} \sum_{n=1}^{\infty} W_{m n} \sin \left(\frac{m \pi x}{a}\right) \sin \left(\frac{n \pi y}{b}\right)$
$\psi_{x}(x, y, t)=\sum_{m=1}^{\infty} \sum_{n=1}^{\infty} \Psi_{x_{m n}} \cos \left(\frac{m \pi x}{a}\right) \sin \left(\frac{n \pi y}{b}\right)$
$\psi_{y}(x, y, t)=\sum_{m=1}^{\infty} \sum_{n=1}^{\infty} \Psi_{y_{m n}} \sin \left(\frac{m \pi x}{a}\right) \cos \left(\frac{n \pi y}{b}\right)$

where $W_{m n}, \Psi_{x_{m n}}$ and $\Psi_{y_{m n}}$ are three generalized coordinates, $m$ and $n$ are mode numbers in $x$ - and $y$ directions respectively.

The transverse normal load $q$ is also expanded as:

$q(x, y, t)=\sum_{m=1}^{\infty} \sum_{n=1}^{\infty} q_{m n}(t) \sin \left(\frac{m \pi x}{a}\right) \sin \left(\frac{n \pi y}{b}\right)$

where $q_{m n}(t)$ is the modal participation term.

Choice of the correct form of the Airy's function $\phi(x, y, t)$ depends on the problem formulation -

- For linear problem involving small strain and small deflection, $\phi(x, y, t)=0$. Hence, $q^{*}=$ 0 and it can be proved that Eq. (15) reduces to a linear FSDT solution.

- In a nonlinear theory (small strain but large deflection), the function $\phi(x, y, t)$ needs to satisfy the edge conditions, namely, immovable or movable edge.

Following the approach of (Nishawala 2011), the Airy's function is defined as:

$$
\begin{aligned}
& \phi(x, y, t)=P_{x} x^{2}+P_{y} y^{2}+ \\
& \sum_{m=1}^{\infty} \sum_{n=1}^{\infty} \phi_{m n}(t) \sin \left(\frac{m \pi x}{a}\right) \sin \left(\frac{n \pi y}{b}\right)
\end{aligned}
$$


where $P_{x}$ and $P_{y}$ are functions of tensile loads that restrain the edges from moving, and $\phi_{m n}(t)$ is the temporal modal terms for Airy's function.

Using the general expressions of strain-displacement relationship, it is able to give the axial displacements $\delta_{x}$ and $\delta_{y}$ along the plate edges $y=[0, b]$, and $x=[0, a]$ as:

$\delta_{x}=\int_{0}^{a}\left[A_{11}^{*} \frac{\partial^{2} \phi}{\partial y^{2}}+A_{12}^{*} \frac{\partial^{2} \phi}{\partial x^{2}}-A_{16}^{*} \frac{\partial^{2} \phi}{\partial x \partial y}-\frac{1}{2}\left(\frac{\partial w}{\partial x}\right)^{2}\right] d x=0($

$\delta_{y}=\int_{0}^{b}\left[A_{12}^{*} \frac{\partial^{2} \phi}{\partial y^{2}}+A_{22}^{*} \frac{\partial^{2} \phi}{\partial x^{2}}-A_{26}^{*} \frac{\partial^{2} \phi}{\partial x \partial y}-\frac{1}{2}\left(\frac{\partial w}{\partial y}\right)^{2}\right] d y=0(25)$

Equations (24) and (25) when solved together with Eqs. (15) and (20) by substituting the solution functions defined in Eqs. $(21-23)$ and the application of the Galerkin's procedure produce nonlinear ordinary differential equations of structure in time domain. To have tractable mathematical expressions, the authors adapted the one-to-one approximation, which has been investigated by (Nishawala 2011). In this approach, only the same modal terms are accounted for when two summation functions are multiplied. This significantly reduces the complexities, giving out the well-known Duffing equation below:

$\ddot{W}_{m n}+\widetilde{K}_{m n} W_{m n}+\tilde{\zeta}_{m n} W_{m n}^{3}=F_{m n}$

where

$\widetilde{K}_{m n}=\frac{\widetilde{D}_{m n}-\widetilde{V}_{m n}}{\rho h \tilde{L}_{m n}}, \tilde{\zeta}_{m n}=\frac{\zeta_{m n}}{\rho h}, F_{m n}=\frac{16 q}{\rho h m n \pi^{2}}$

Together with the initial conditions, Eq. (26) can be solved using the same procedure (NSFD) presented in subsection 3.1. For brevity, these procedures will not be repeated here.

\subsubsection{In-water}

Coupling with $\mathrm{DAA}_{1}$ for the air-backed condition has been presented in detail by (Sone Oo et al. 2020b, 2021). To entail the purpose of this paper, only a brief summary of the derivation is presented here.

We use the same structural equation shown in Eq. (26). However, the force function is redefined as:

$F_{m n}(t)=\iint_{S}\left[P_{i}(t)+P_{s_{L}}(x, y, t)-P_{s_{R}}(x, y, t)\right] \alpha_{m n} d S$

where $\alpha_{m n}=\sin \left(\frac{m \pi x}{a}\right) \sin \left(\frac{n \pi y}{b}\right)$, and $d S=d x d y$. Note that the incident pressure is supposed to be evenly distributed across the plate and thus, does not depend on spatial coordinates. The scattered pressures for both left and right sides of the plate are functions of spatial and temporal variables, taking the following forms:

$P_{s_{L}}(x, y, t)=\sum_{m=1}^{\infty} \sum_{n=1}^{\infty} p_{m n}^{L}(t) \alpha_{m n}(x, y)$

$P_{S_{R}}(x, y, t)=\sum_{m=1}^{\infty} \sum_{n=1}^{\infty} p_{m n}^{R}(t) \alpha_{m n}(x, y)$

where $p_{m n}^{L}(t)$ and $p_{m n}^{R}(t)$ are modal terms.

By substituting Eqs. (29a - 29b) to Eq. (28), and using the orthogonality condition, we get:

$F_{m n}(t)=\left(\frac{A_{f}}{4}\right)\left[\left(\frac{16}{m n \pi^{2}}\right) P_{i}(t)+p_{m n}(t)\right]$
$\mathrm{DAA}_{1}$ equation for the $2 \mathrm{D}$ deformable plate after applying the Galerkin's procedure can be given as:

$p_{m n}^{L}=-D_{f_{m n}} p_{m n}^{L}-\rho_{w} c_{w} \dot{V}_{m n}+\left(\frac{16}{m n \pi^{2}}\right) \dot{P}_{i}$

$p_{m n}^{R}=-D_{f_{m n}} p_{m n}^{L}+\rho_{w} c_{w} \dot{V}_{m n}$

in which the difference is the presence of the incident particle acceleration on the left side of the plate. Note that $D_{f_{m n}}=\rho_{w} c_{w} / M_{a_{m n}}$ needs to be calculated for each mode $m, n$. The modified formulation of (Greenspon 1961)'s water-added mass per unit area is (also see, Sone Oo et al. 2021):

$M_{a_{m n}}=\frac{1}{2} \rho_{w} b f\left(\frac{a}{b}\right) \sum_{j=1}^{\infty} \frac{64}{m n j^{2} \pi^{4}}$

where $j=1,3,5, \ldots$.

Equation (32) can be solved using NSFD method:

$p_{m n}^{L}{ }^{i+1}=p_{m n}^{L}{ }^{i} e^{-D_{f m n} \Delta t}+\left(\frac{1-e^{-D_{f m n} \Delta t}}{D_{f m n}}\right)\left(-\rho_{w} c_{w} \dot{V}_{m n}^{i}+\right.$

$\left.\dot{P}_{i}^{i}\left(\frac{16}{m n \pi^{2}}\right)\right)$

$p_{m n}^{R}{ }^{i+1}=\varrho\left[p_{m n}^{R}{ }^{i} e^{-D_{f m n} \Delta t}+\left(\frac{1-e^{-D_{f m n} \Delta t}}{D_{f m n}}\right)\left(\rho_{w} c_{w} \dot{V}_{m n}^{i}\right)\right]$

Recall that $\varrho$ is a flag to change from water-backed to air-backed condition.

The expressions for the displacement and velocity are the same as the ones shown in Eqs. (9) and the acceleration as:

$\dot{V}_{m n}^{i+1}=-\omega_{m n}^{2}{ }^{i} W_{m n}^{i}+\frac{4}{M_{s}} F_{m n}^{i}$

where $M_{s}=\rho h A_{f}$ is the mass of the plate. Note also that for linear theory, the angular frequency $\omega_{m n}=$ $\sqrt{K_{M n} / m_{s}}$ is the same for all time steps whereas in the nonlinear theory, the frequency changes due to local linearization since:

$\omega_{m n}^{i}=\sqrt{\widetilde{K}_{m n}+\tilde{\zeta}_{m n}\left(W_{m n}^{i}\right)^{2}}$

where the initial conditions are taken as:

$W_{m n}(0)=V_{m n}(0)=0, \dot{V}_{m n}(0)=2 P_{0} / m_{s}, p_{m n}^{L}(0)=$

$16 P_{0} /\left(m n \pi^{2}\right), p_{m n}^{R}(0)=0$

\section{RESULTS \& DISCUSSIONS}

In this section, three types of problems are studied: (1) spring-supported rigid plate subjected to a plane shock exponential wave, (2) simply-supported steel plate subjected to a uniformly distributed suddenly applied pressure load, and (3) simply-supported composite plate subjected to an exponentially decaying plane shock wave. The results are compared with numerical solutions from LS-DYNA/USA (DAA $\left.{ }_{1}\right)$ without cavitation or damage. The Finite Element (FE) models are detailed in each corresponding subsection. The effect of geometric nonlinearity is also investigated by using an air-backed composite plate subjected to various step loadings. 


\subsection{Case study 1: spring-supported rigid plate under plane shock loading}

A rigid square plate with dimensions $(269.8 \mathrm{~mm} \mathrm{x}$ $269.8 \mathrm{~mm}$ ), thickness $6.35 \mathrm{~mm}$, density $7800 \mathrm{~kg} \cdot \mathrm{m}^{-3}$, and natural frequency $100 \mathrm{~Hz}$ is subjected to a plane shock pressure generated at $15.24 \mathrm{~m}$ from an explosion of $136 \mathrm{~kg}$ of T.N.T (Taylor 1941). The associated peak pressure and decay time are $15.4 \mathrm{MPa}$ and $0.435 \mathrm{~ms}$ respectively. The plate is submerged in water with density $1000 \mathrm{~kg} \cdot \mathrm{m}^{-3}$ and acoustic speed $1400 \mathrm{~m} \cdot \mathrm{s}^{-1}$.

In LS-DYNA/USA, a single finite element platespring model, shown in Figure 1, is constructed. Instead of modelling the fluid explicitly, however, $\mathrm{DAA}_{1}$ boundary elements are prescribed over the surface of the plate. Rigid material definition is used. Cavitation or hydrostatic pressure is not accounted for. Water-backed condition can be invoked in LSDYNA/USA (DAA ${ }_{1}$ ) by specifying the water-backed node numbers. Same value of water-added mass $\left(M_{a} / M_{s}=3.056\right)$ is used in both numerical and semi-analytical approaches.

Comparisons of the results for air- and waterbacked conditions are shown in Figure 3. The maximum response of the water-backed plate is found to be about $24 \%$ less than that of the air-backed plate. The total pressure profiles look similar between the two conditions. The displacement is normalized by $W_{m}=$ $2 P_{0} \tau /\left(\rho_{w} c_{w}\right)$, maximum displacement given by Taylor's theory in the air-backed condition. The normalization of time is done by dividing by $T_{d}=a /\left(2 c_{w}\right)$, which is the diffraction time of the plate. It is observed that the NSFD method gives almost identical results compared to LS-DYNA/USA $\left(\mathrm{DAA}_{1}\right)$.

\subsection{Case study 2: simply-supported steel plate under step loading}

A simply-supported (immovable edge) steel plate (size: $100 \mathrm{~mm}$ x $100 \mathrm{~mm}$, thickness: $5.76 \mathrm{~mm}$, density: $7800 \mathrm{~kg} \cdot \mathrm{m}^{-3}$, Young's modulus: $200 \mathrm{GPa}$, Poisson's ratio: 0.3 ) is subjected to uniformly distributed step loading of pressure $P_{0}=2.5 \mathrm{MPa}$. Cavitation is not considered in the analysis. The acoustic properties of water are taken as $\rho_{w}=1025 \mathrm{~kg} \cdot \mathrm{m}^{-3}$ and $c_{w}=$ $1500 \mathrm{~m} . \mathrm{s}^{-1}$. Failure is not considered as well. A quarter plate model $(50 \mathrm{~mm} \times 50 \mathrm{~mm})$ is constructed in LS-DYNA using a total of 169 fully-integrated shell elements along with an elastic material. Five throughthickness integration points are given. A shear correction factor of 5/6 is applied. Air- and water-backed conditions are defined through USA input cards.

Figure 4 compares the time histories of the deflection and stress in $x$-direction at the center of the steel plate. It can be seen that not only the central deflection but also the stress agrees well $(<5 \%$ discrepancy relative to analytical results) between the two approaches considered in this paper. Compared to the air-backed plate, the water-backed plate response shows a slight

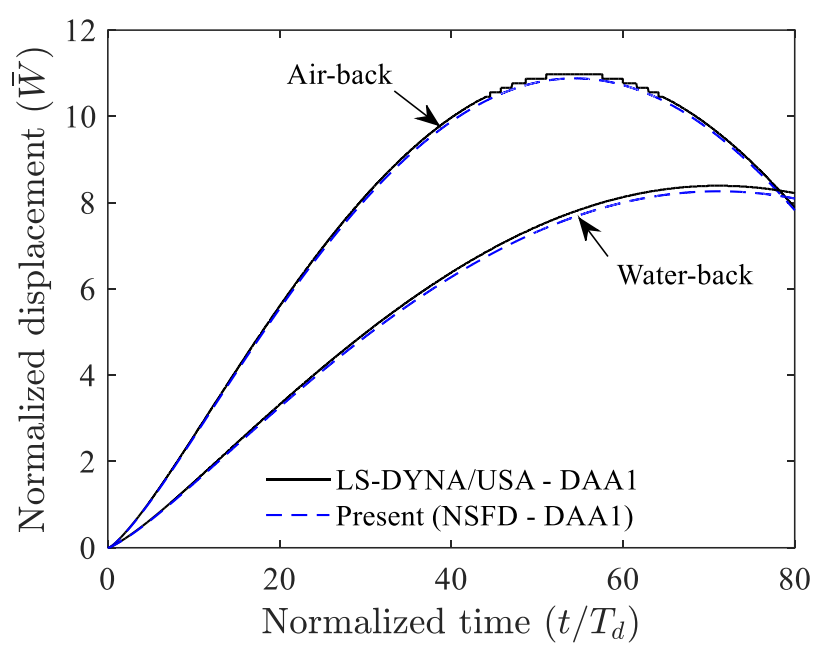

(a) Normalized displacement Vs normalized time

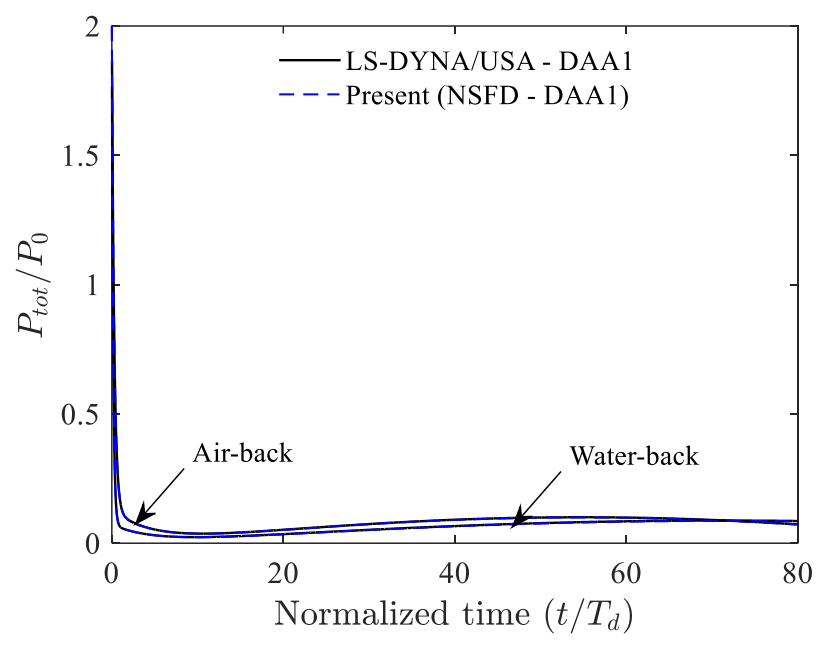

(b) Normalized total pressure Vs normalized time

Figure 3. UNDEX responses of spring-supported rigid plate in air- and water-backed conditions

decrease $(\approx 24 \%)$ in amplitude and an increase in the plate response time due to an additional involvement of water-added mass at the back of the plate. It should be noted that the plasticity effect has not been considered in this study and so, the case study is useful strictly to verify the mathematical relevance of the model compared to LS-DYNA/USA (DAA 1$)$ approach.

\subsection{Case study 3: simply-supported CFRP plate under plane shock loading}

Case 3 considers a simply-supported (immovable edge) carbon-fiber/epoxy (CFRP) plate with the same dimension and thickness as case study 2. A material density of $1548 \mathrm{~kg} \cdot \mathrm{m}^{-3}$ and a stacking sequence of $[ \pm 45 / 0 / 0 / 0 / \pm 45 / 0 / 0 / 0 / 90 / 90]_{S}$ are used with mechanical characteristics defined in Table 1. Only a quarter of the plate is modeled in LS-DYNA by using symmetric boundary conditions. Modified fully-integrated shell elements (EQ: -16) along with the composite material (MAT 54) are employed. The peak 


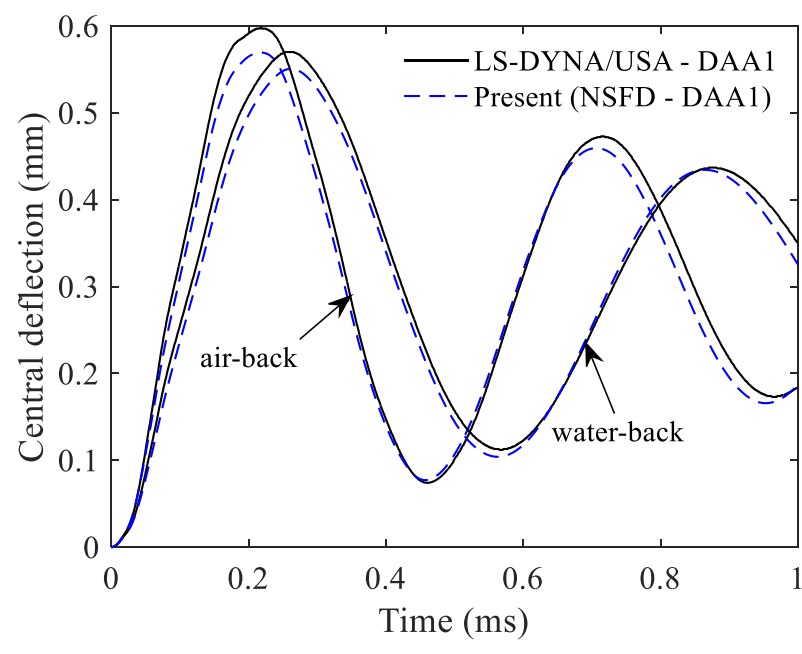

(a) Central deflection - time history

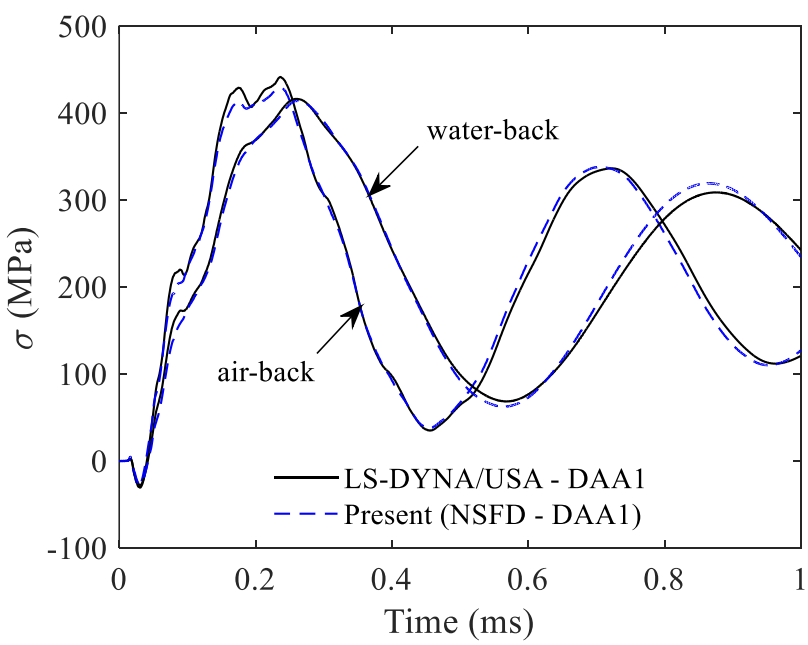

(b) Central stress - time history

Figure 4. Time histories of simply-supported rectangular steel plate responses in air- and water-backed conditions

pressure of 1.5 MPa and load decay time of $1.3 \mathrm{~ms}$ corresponding to a $586 \mathrm{~kg}$ of T.N.T explosive detonated at $169 \mathrm{~m}$ stand-off distance (shock factor $=$ 0.14 ) are considered.

Figure 5 shows time evolutions of the CFRP plate deflection and stress (in fiber direction) at bottom ply and center of the plate when subjected to UNDEX. The central deflection shows $8 \%$ discrepancy with respect to the analytical results whereas the stress shows less than $15 \%$ discrepancy relative to the analytical values (with $x$-y displacements) for both air- and waterbacked cases. This is mainly due to the possible involvement of the in-plane stretching. By restraining the translational displacements in LS-DYNA/USA $\left(\mathrm{DAA}_{1}\right)$, it can be seen in Figure 5(b) that the analytical and numerical stress results become much closer. Assessment to the mid-plane stretching also reveals that stretching amounts to about $13 \%$ of maximum bending stress in this case. Such in-plane stretching effects may, therefore, need to be considered in the future.
Table 1. Characteristics of the carbon fiber/epoxy lamina

\begin{tabular}{llll}
$E_{11}(\mathrm{GPa})$ & 138 & $E_{22}=E_{33}(\mathrm{GPa})$ & 8.98 \\
$v_{12}=v_{13}$ & 0.281 & $v_{23}$ & 0.385 \\
$G_{12}=G_{13}(\mathrm{GPa})$ & 3.66 & $G_{23}(\mathrm{GPa})$ & 3.24 \\
\hline
\end{tabular}

*Taken from quasi-static tests performed by the authors

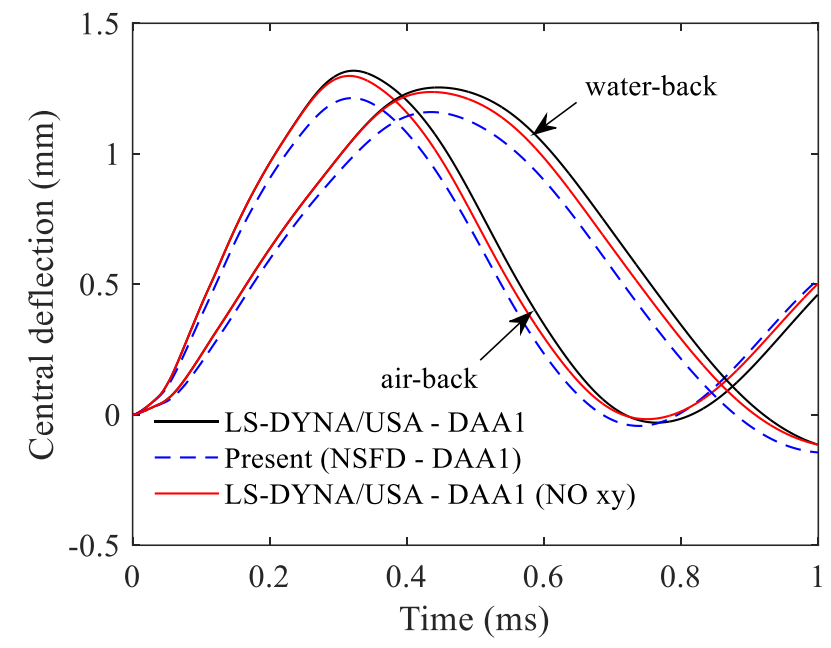

(a) Central deflection - time history

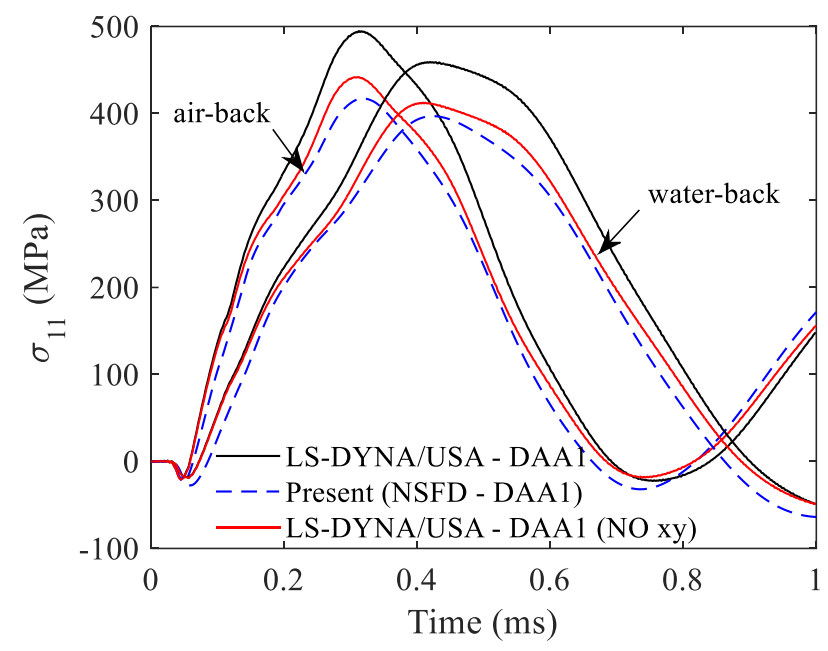

(b) Central stress (bottom ply) - time history

Figure 5. Time histories of simply-supported rectangular CFRP plate responses in air- and water-backed conditions

\subsection{Geometric nonlinear effect}

A geometric nonlinear effect due to large deflection is investigated by using a flexible CFRP plate with larger length-thickness ratio, $a / h=69.4$. Only airbacked results are shown here since the conclusion is the same for the water-backed cases. Step loading with varying levels of peak pressures is considered. Linear analytical solutions are also plotted here to compare. In Figure 6, the improvement after incorporating the geometric nonlinear effects (after $W_{\text {max }} / h \geq 0.4$ ) can be clearly observed. Figure 7 shows comparison of the central deflection - time histories evaluated by analytical (linear), analytical (nonlinear) and numerical approaches when the step loading of $0.1 \mathrm{MPa}$ is applied. It can be seen that the 


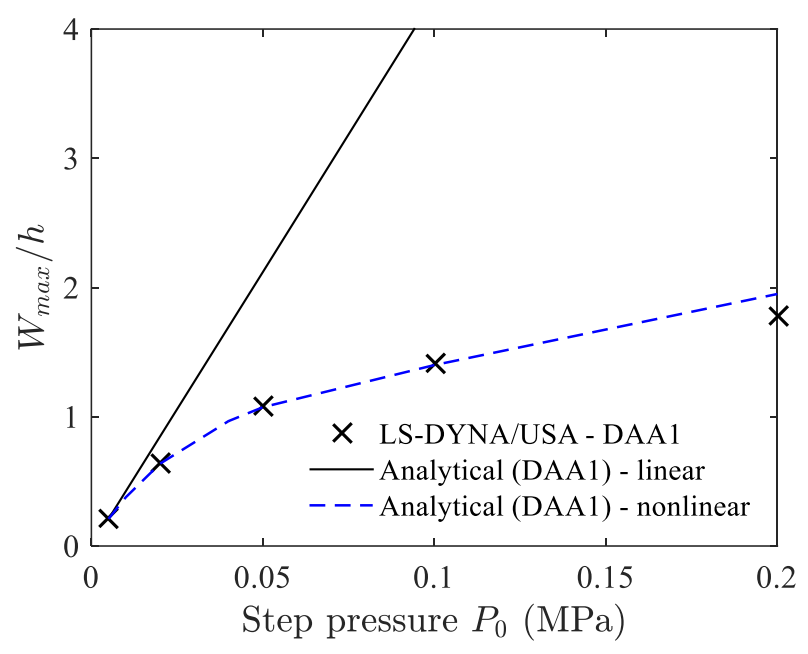

Figure 6. Nonlinear UNDEX responses of simply-supported rectangular CFRP plate in an air-backed condition

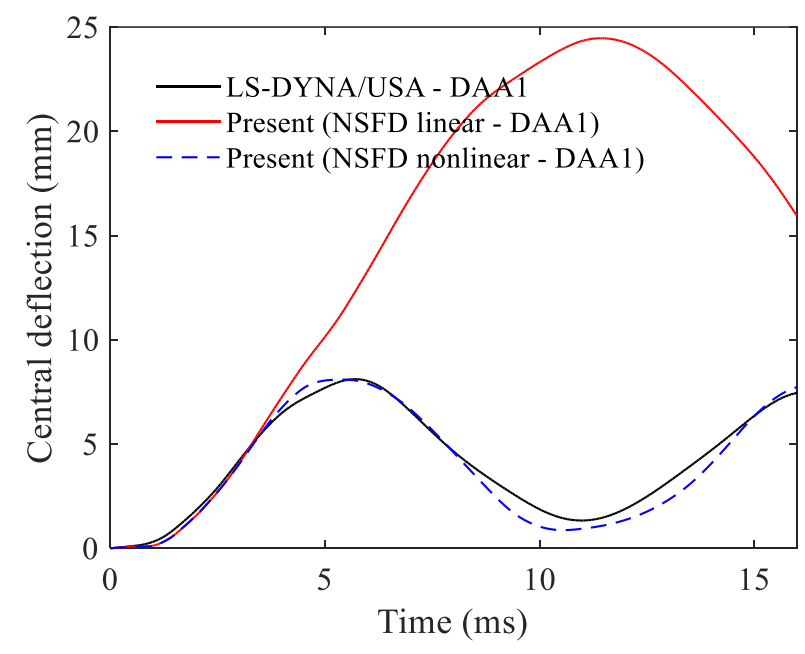

Figure 7. Comparison between linear and nonlinear responses of CFRP plate $\left(\boldsymbol{P}_{\mathbf{0}}=\mathbf{0 . 1} \mathrm{MPa}\right)$

linear FSDT-DAA 1 model is only comparable until about $4 \mathrm{~ms}$. Geometric nonlinearity is found to decrease period of oscillation as well as the amplitude of the dynamic response.

\section{CONCLUSION \& PERSPECTIVES}

A semi-analytical design tool is introduced to determine the response of air-backed and water-backed composite plates under a far-field underwater explosion. The analytical development is presented for both the rigid plate-spring system and simply-supported deformable plates. It has been shown that the present semi-analytical formulations for deformable plates obtain close agreement $(<10 \%$ for deflection and $<15 \%$ for stress) compared to numerical solutions using LS-DYNA/USA (DAA $\left.{ }_{1}\right)$. The nonlinear behavior is also captured by the consideration of the one-to-one approximation and the quadratic straindisplacement relationships.
On characterizing the incident load, nevertheless, only a simple plane shock wave associated with a farfield underwater explosion is adapted. This is indeed an idealized assumption to simplify the case study. In the future, a spherical wave as well as the possible contribution of the oscillating gas bubble should be examined. Moreover, the analysis is performed only within elastic regime with the intention of developing a semi-analytical design tool for the rapid analysis of composite plate response. From an industrial point of view, the present semi-analytical model could be used to determine the maximum deflection and the maximum stress especially in the preliminary design stage.

As part of the future work, it should be investigated in more details about the influence of stretching due to in-plane displacements as well as the rotatory inertia effect. The applicability of the one-to-one approximation is seen to be working well to predict the deflections but should further be explored in determining the stresses since those functions contain second-order differentiations. Correct predictions of the stresses would help the designers predict the damage initiation correctly. Cavitation, hydrostatic pressure, structural damping and post-damage effects are disregarded at the moment and so, should be studied in the future. Finally, the approach presented in this paper should be compared with a fully-coupled numerical simulation such as LS-DYNA/USA involving acoustic volume elements or with the experiments for various scenarios and plate aspect ratios. These perspectives would be taken into account in the subsequent publications.

\section{APPENDIX}

$$
\begin{aligned}
& l_{1}=s_{4} s_{7}-s_{1} s_{10}, l_{2}=s_{4} s_{8}+s_{5} s_{7}-s_{1} s_{11}-s_{2} s_{10}, l_{3}= \\
& s_{4} s_{9}+s_{5} s_{8}+s_{6} s_{7}-s_{1} s_{12}-s_{2} s_{11}-s_{3} s_{10}, l_{4}=s_{5} s_{9}+ \\
& s_{6} s_{8}-s_{2} s_{12}-s_{3} s_{11}, l_{5}=s_{6} s_{9}-s_{3} s_{12}, l_{6}=s_{1}+s_{10}, l_{7}= \\
& s_{2}+s_{10}, l_{7}=s_{2}+s_{11}, l_{8}=s_{3}+s_{12} \\
& {[s]=\frac{T_{s}}{K_{s}}\left[A_{s}^{*}\right]\left[D_{126}\right]}
\end{aligned}
$$

where $T_{S}$ is a tracing constant for transverse shear effect $\left(T_{s}=0\right.$ if transverse shear effect is neglected), and $K_{s}$ is the shear correction factor. And:

$$
\begin{aligned}
& {[s]=\left[\begin{array}{llllllllllll}
s_{1} & s_{2} & s_{3} & s_{4} & s_{5} & s_{6} & s_{7} & s_{8} & s_{9} & s_{10} & s_{11} & s_{12}
\end{array}\right]^{T}} \\
& {\left[D_{126}\right]=\left[\begin{array}{l}
D_{11} \\
D_{12} \\
D_{16} \\
D_{22} \\
D_{26} \\
D_{66}
\end{array}\right],\left[A_{S}^{*}\right]=\left[\begin{array}{cccccc}
A_{55}^{*} & 0 & A_{45}^{*} & 0 & 0 & 0 \\
0 & A_{45}^{*} & 2 A_{55}^{*} & 0 & 0 & A_{45}^{*} \\
0 & 0 & 0 & 0 & A_{45}^{*} & A_{55}^{*} \\
0 & 0 & A_{55}^{*} & 0 & 0 & A_{45}^{*} \\
0 & A_{55}^{*} & 0 & 0 & 2 A_{45}^{*} & A_{55}^{*} \\
0 & 0 & 0 & A_{45}^{*} & A_{55}^{*} & 0 \\
A_{45}^{*} & 0 & A_{44}^{*} & 0 & 0 & 0 \\
0 & A_{44}^{*} & 2 A_{45}^{*} & 0 & 0 & A_{44}^{*} \\
0 & 0 & 0 & 0 & A_{44}^{*} & A_{45}^{*} \\
0 & 0 & A_{45}^{*} & 0 & 0 & A_{44}^{*} \\
0 & A_{45}^{*} & 0 & 0 & 2 A_{44}^{*} & A_{45}^{*} \\
0 & 0 & 0 & A_{44}^{*} & A_{45}^{*} & 0
\end{array}\right]}
\end{aligned}
$$




$$
\begin{gathered}
{[\bar{s}]=\left[\begin{array}{ccc}
-s_{10} & 0 & s_{7} \\
s_{4}-s_{11} & s_{7} & s_{8}-s_{1}-3 s_{10} \\
s_{5}-s_{12} & s_{8}-s_{1}-s_{10} & s_{9}-s_{2}+3\left(s_{4}-s_{11}\right) \\
s_{6} & s_{4}+s_{9}-s_{11}-s_{12} & 3\left(s_{5}-s_{12}\right)-s_{3} \\
0 & s_{5}-s_{3}-s_{12} & 3 s_{6} \\
0 & s_{6} & 0 \\
0 & 0 & 0
\end{array}\right] \text { (A5) }} \\
0 \\
0
\end{gathered}
$$

\section{ACKNOWLEDGEMENT}

This research work has been financially supported by DGA-DGE, under grant number 172906080. The authors would like to thank Calcul-Meca and Multiplast companies for their technical support.

\section{REFERENCES}

Arora, H., Rolfe E., Kelly M., and Dear, J.P. 2017. "FullScale Air and Underwater- Blast Loading of Composite Sandwich Panels." In Explosion Blast Response of Composites, eds. Adrian P Mouritz and Yapa D.S. Rajapakse. Woodhead Publishing, 16199.

Barras, G. 2012. "Interaction Fluide-Structure: Application Aux Explosions Sous-Marines En Champ Proche." University of Sciences and Technologies, Lille, France.

Brochard, K., Le Sourne, H., and Barras, G. 2018. "Extension of the String-on-Foundation Method to Study the Shock Wave Response of an Immersed Cylinder." International Journal of Impact Engineering 117 (May 2017): 138-52. https://doi.org/10.1016/j.ijimpeng.2018.03.007.

Brochard, K., Le Sourne, H., and Barras, G. 2020. "Estimation of the Response of a Deeply Immersed Cylinder to the Shock Wave Generated by an Underwater Explosion." Marine Structures 72 (January).

Cole, R.H. 1948. Underwater Explosions. Princeton: Princeton University Press.

Dear, J.P., Rolfe, E., Kelly, M., Arora, H., and Hooper, P.A. 2017. "Blast Performance of Composite Sandwich Structures." Procedia Engineering 173: 471-78. www.elsevier.com/locate/procedia.

DeRuntz, J.A. Jr. 1989. "The Underwater Shock Analysis Code and Its Applications." In Proceedings of the $60^{\text {th }}$ Shock and Vibration Symposium, 89-107.

Felippa, C.A., and DeRuntz, J.A. 1984. "Finite Element Analysis of Shock-Induced Hull Cavitation." Computer Methods in Applied Mechanics and Engineering 44: 297-337.

Geers, T.L. 1978. "Doubly Asymptotic Approximations for Transient Motions of Submerged Structures." The Journal of the Acoustical Society of America 64: $1500-1508$.

Greenspon, J.E. 1961. "Vibrations of Cross-Stiffened and Sandwich Plates with Application to Underwater Sound Radiators." The Journal of the Acoustical Society of America 33(11): 1485-97.

Hall, D.J. 1989. "Examination of the Effects of Underwater Blasts on Sandwich Composite Structures." Composite Structures 11(2): 101-20.

Hoo Fatt, M.S., and Dushyanth Sirivolu. 2017. "Marine Composite Sandwich Plates under Air and Water Blasts." Marine Structures 56: 163-85. http://dx.doi.org/10.1016/j.marstruc.2017.08.004.

Kármán, Th. V. 1907. "Festigkeitsprobleme Im Maschinenbau." In ENCYKLOPÄDIE DER MATHEMATISCHEN WISSENSCHAFTEN, ed. Müller C. Klein F. Vieweg+Teubner Verlag, Wiesbaden, 311-85.

Keil, A.H. 1961. "The Response of Ships to Underwater Explosions." In Annual Meeting, New York, N.Y.: The Society of Naval Architects and Marine Engineers, 366-410.

Klenow, B., and Brown, A. 2010. "Prevention of Pressure Oscillations in Modeling a Cavitating Acoustic Fluid." Shock and Vibration 17(2): 137-59.

Liu, Z., and Yin L. Young. 2008. "Transient Response of Submerged Plates Subject to Underwater Shock Loading: An Analytical Perspective." Journal of Applied Mechanics - Transactions of the ASME 75.

Mair, H.U. 1999. "Review: Hydrocodes for Structural Response to Underwater Explosions." Shock and Vibration 6(2): 81-96.

Mei, C., and Prasad, C.B. 1989. "Effects of Large Deflection and Transverse Shear on Response of Rectangular Symmetric Composite Laminates Subjected to Acoustic Excitation." Journal of Composite Materials 23(6): 606-39.

Mouritz, A.P., Gellert, E., Burchill, P., and Challis, K. 2001. "Review of Advanced Composite Structures for Naval Ships and Submarines." Composite Structures 53(1): 21-41.

Mouritz, A.P. 2017. "Progress Toward Explosive BlastResistant Naval Composites." In Explosion Blast Response of Composites, eds. Adrian P Mouritz and Yapa D.S. Rajapakse. Woodhead Publishing, 375408. http://dx.doi.org/10.1016/B978-0-08-1020920.00014-5\%0ACopyright.

Newton, R.E. 1978. Effects of Cavitation on Underwater Shock Loading - Part I. Washington, D.C.: Defense Nuclear Agency. http://hdl.handle.net/10945/29265.

Nishawala, V. 2011. "Study of Large Deflection of Beams and Plates." University of New Jersey.

Office of Naval Research. 1950. 1 Underwater Explosion Research: A Compendium of British and American Reports. eds. G.K. Hartmann and E.G. Hill. Washington, D.C.: Office of Naval Research, Dept. of the Navy.

Porfiri, M., and Gupta, N. 2009. "A Review of Research on Impulsive Loading of Marine Composites." In Major Accomplishments in Composite Materials and Sandwich Structures: An Anthology of ONR Sponsored Research, eds. Daniel I.M., Y.D.S. Rajapakse, and E.E. Gdoutos. Springer Science + 
Business Media B.V., 169-94. www.springer.com.

Schiffer, A., and Tagarielli, V.L. 2012. "The Response of

Rigid Plates to Blast in Deep Water: Fluid-Structure Interaction Experiments." Proceedings of the Royal Society A: Mathematical, Physical and Engineering Sciences 468(2145): 2807-28.

Shin, Y.S. 2004. "Ship Shock Modeling and Simulation for Far-Field Underwater Explosion." Computers and Structures 82(23-26): 2211-19.

Sigrist, J.F. 2015. Fluid-Structure Interaction: An Introduction to Finite Element Coupling. DCNS Research, France: John Wiley \& Sons Limited Registered.

Sone Oo, Y. P., Le Sourne, H., and Dorival, O. 2020a. “On the Applicability of Taylor's Theory to the Underwater Blast Response of Composite Plates." International Journal of Impact Engineering 145(July): $\quad$ 1-15. https://doi.org/10.1016/j.ijimpeng.2020.103677.

Sone Oo, Y.P., Le Sourne, H. and Dorival, O. 2019. "Development of Analytical Formulae to Determine the Response of Submerged Composite Plates Subjected to Underwater Explosion." In The 14th International Symposium on Practical Design of Ships and Other Floating Structures - PRADS 2019, Yokohama, Japan, 1-21.

Sone Oo, Y.P., Le Sourne, H., and Dorival, O. 2020b. "Coupling of First-Order Doubly Asymptotic Approximation to Determine the Response of Orthotropic Plates Subjected to an Underwater Explosion." In International Conference on Ships and Offshore Structures - ICSOS 2020, ed. Prof. Sören Ehlers. University of Strathclyde, Glasgow, UK.

Sone Oo, Y.P., Le Sourne, H., and Dorival, O. 2021. “A coupled closed-form/Doubly Asymptotic Approximation approach for the response of orthotropic plates subjected to an underwater explosion". Ships and Offshore Structures. https://doi.org/10.1080/17445302.2021.1918962

Songolo, M.E., and Bidégaray-Fesquet, B. 2018. "Nonstandard Finite-Difference Schemes for the Two-Level Bloch Model." International Journal of Modeling, Simulation, and Scientific Computing 9(4): 1-23.

Le Sourne, H., Besnier, F. and Couty, N. 2003. "LSDYNA Applications in Shipbuilding." $4^{\text {th }}$ European LS-DYNA Users Conference: 1-16.

Taylor, G.I. 1941. "The Pressure and Impulse of Submarine Explosion Waves on Plates." In The Scientific Papers of G. I. Taylor, Vol. III, Cambridge, UK: Cambridge University Press, 287-303. 